\title{
The Lasting Effect of Words on Feelings: Words May Facilitate Exposure Effects to Threatening Images
}

\author{
Golnaz Tabibnia, Matthew D. Lieberman, and Michelle G. Craske \\ University of California, Los Angeles
}

\begin{abstract}
Previous studies have shown that mere words, particularly affective words, can dampen emotional responses. However, the effect of affective labels on emotional responding in the long term is unknown. The authors examined whether repeated exposure to aversive images would lead to more reduction in autonomic reactivity a week later if the images were exposed with single-word labels than without labels. In Experiment 1, healthy individuals were exposed to pictures of disturbing scenes with or without labels on Day 1. On Day 8, the same pictures from the previous week were exposed, this time without labels. In Experiment 2, participants were spider fearful and were exposed to pictures of spiders. In both experiments, although repeated exposure to aversive images (without labels) led to long-term attenuation of autonomic reactivity, exposure plus affective labels, but not nonaffective labels, led to more attenuation than exposure alone. Thus, affective labels may help dampen emotional reactivity in both the short and long terms. Implications for exposure therapy and translational studies are discussed.
\end{abstract}

Keywords: fear, psychophysiology, emotion regulation, exposure, language

Supplemental materials: http://dx.doi.org/10.1037/1040-3590.8.3.307

The typical treatment for phobias and other anxiety disorders involves repeated exposure to the feared situation or stimulus so that the fear or anxiety may extinguish over time. Although exposure therapy is an established treatment for phobias (Chambless \& Hollon, 1998), methods that can improve outcome of exposure therapy are still needed (Craske, 1999; Craske \& Mystkowski, 2006). The implementation of exposure therapy for treatment of anxiety and phobias in humans has been in large part influenced by experimental findings of Pavlovian associative learning and extinction in nonhuman animals (Eysenck, 1979; Pavlov, 1927). However, as many critics of conditioning theories of human anxiety have asserted (e.g., Brewer, 1974), human learning and emotional processing are more complex than in other animals, and simple conditioning accounts cannot fully explain fear and anxiety in humans. Importantly, humans often use language to regulate

Golnaz Tabibnia, Department of Psychiatry and Behavioral Sciences, University of California, Los Angeles; Matthew D. Lieberman and Michelle G. Craske, Department of Psychology, University of California, Los Angeles.

This work was supported by a grant (IR21 MG65066-01) from the National Institute of Mental Health to Michelle G. Craske and a predoctoral National Research Service Award (MH 070146-01A1) from the National Institute of Mental Health to Golnaz Tabibnia. We thank Dr. Bruce Naliboff for help with the psychophysiology equipment. We would also like to thank the dedicated research assistants for help with stimulus preparation and data collection, including Suzan Berghoudian, Chris Prodoehl, Mara Zulauf, Gizel Tabibnia, Yenni Arredondo, Chau Mai, Jane Chiu, Brittany Meyer-Belnap, and especially Tristen Inagaki. Do not cite without permission.

Correspondence concerning this article should be addressed to Golnaz Tabibnia, Department of Psychology, Franz Hall, University of California, Los Angeles, Los Angeles, CA 90095-1563. E-mail: golnaz@ucla.edu emotions, and the use of language has been shown to affect learned fear in ways that cannot be tested in nonhuman animals (Davey, 1992).

The idea that verbalization of feelings can help reduce distress is not new in psychology (e.g., Titchener, 1908). Several empirical studies have now demonstrated that verbal disclosure of a traumatic experience can improve physical and psychological wellbeing in the long-term (Hemenover, 2003; Pennebaker, 1997). Recent studies using functional magnetic resonance imaging (fMRI) also indicate a benefit of linguistic processing of aversive experience, at least in the short term (Lieberman, Eisenberger, Crockett, Tom, Pfeifer, \& Way, 2007). When participants are instructed to reduce their internal emotional experience while looking at evocative pictures or films, emotional response to those stimuli decreases during self-regulation, as indicated by self-report (Gross, 1998) and by decreased activity in amygdala (Beauregard, Levesque, \& Bourgouin, 2001; Levesque et al., 2003; Ochsner, Bunge, Gross, \& Gabrieli, 2002), the part of the brain most commonly associated with fear and anxiety processes. Although it is not clear what cognitive strategies people use in downregulating their negative emotional experience, those strategies may involve some form of internal verbal thought. Interestingly, even mere linguistic processing of evocative pictures, without any explicit instruction to self-regulate, also leads to smaller amygdala responses (Critchley et al., 2000; Hariri, Bookheimer, \& Mazziotta, 2000; Hariri, Mattay, Tessitore, Fera, \& Weinberger, 2003; Lieberman, Eisenberger et al., 2007; Lieberman, Hariri, Jarcho, Eisenberger, \& Bookheimer, 2005) and reduced autonomic reactivity (Hariri et al., 2003; Lieberman, Crockett et al., 2007).

The mechanism underlying downregulation of negative emotions seems to be similar across these studies and involves the prefrontal cortex. Several fMRI studies have suggested that as 
activity in the ventrolateral prefrontal cortex increases, activity in affective regions such as amygdala decreases (Hariri et al., 2000, 2003; Lieberman et al., 2005; Lieberman, Eisenberger et al., 2007; Ochsner et al., 2002). For example, viewing an emotionally evocative picture, such as a fearful face, activates amygdala, whereas viewing the picture along with an affective label, such as the word fearful, activates right ventrolateral prefrontal cortex (VLPFC) but not amygdala. This VLPFC activity is inversely correlated with level of activity in amygdala, suggesting the possibility that language-related prefrontal activity inhibits emotion-related amygdala activity. Importantly, viewing the picture along with a nonaffective label, such as a gender-appropriate name ("John"), activates amygdala as much as viewing pictures alone and does not activate the VLPFC (Lieberman, Eisenberger et al., 2007). Thus, any potential inhibitory effect of words on amygdala may be specific to affective words, rather than labeling per se or the cognitive demands of the task.

If language-related prefrontal activity indeed has an inhibitory effect on amygdala and autonomic reactivity, incorporation of language into exposure therapy for phobia may help improve treatment outcome. The aim of the current experiments was to determine whether emotional responding to aversive stimuli would be attenuated more if those stimuli had been previously exposed along with affective words compared to if they had been previously exposed with nonaffective words or without words.

One issue of combining affective words with exposure treatment is that the words may actually serve as distractors and interfere with adaptive emotional processing (Rachman, 1980). Specifically, it has been suggested that exposure outcome is enhanced when a complete physiological response to the feared stimulus occurs initially during exposure (Foa \& Kozak, 1986; Foa \& McNally, 1996). Therefore, if an affective word is presented before or simultaneously with the evocative stimulus, extinction may be prevented, because the emotional response to the picture may be inhibited before it can begin (see Borkovec, Ray, \& Stoeber, 1998). However, if the word is presented after the evocative stimulus, then an emotional response can be expressed before it is inhibited. In order to reduce emotional reactivity more effectively in the long term, in the current experiments the word was presented after, rather than concurrently with, the evocative stimulus.

To investigate the effect of words on emotional responding in the long term, we employed a paradigm that is a rudimentary analogue of exposure treatment. In two separate experiments, we examined whether repeated exposure to aversive pictures would lead to better attenuation of autonomic reactivity to those pictures a week later if the exposures were accompanied by linguistic processing. In Experiment 1, this effect was investigated in healthy individuals who were exposed to aversive pictures from the International Affective Picture Set (IAPS; Center for the Study of Emotion and Attention [CSEA], 1999; P. J. Lang, Bradley, \& Cuthbert, 1999). We explored the clinical implications of this effect in Experiment 2, in which participants were fearful of spiders and were exposed to pictures of spiders. Autonomic reactivity was determined by skin conductance response (SCR), a measure of sympathetic arousal, and heart rate (HR), a reflection of both sympathetic and parasympathetic activity (Berntson, Cacioppo, \& Quigley, 1993; Öhman, Hamm, \& Hugdahl, 2000). We hypothesized that reactivity would decrease from Day 1 to Day 8 in each condition, but that on Day 8 reactivity would be more reduced in the affective-label conditions compared to the no-label condition.

\section{Experiment 1: Nonselected Participants}

\section{Method}

\section{Participants}

Twenty-seven healthy college students ( 23 females, average age 18.9), enrolled in an introductory psychology class, participated in this study. All participants gave informed consent. Individuals with heart, respiratory, or neurological problems were excluded, as problems of this nature may interfere with autonomic recordings. Similarly, those on psychotropic medications or any other medications that affect autonomic state were excluded. Participants who had a history of fainting at the sight of blood in pictures or movies were also excluded. There was no effect of gender on psychophysiology measures, and hence gender is not discussed further.

\section{Design}

In this within-subjects study, there were four conditions in the exposure session (Day 1). As illustrated in Figure 1A, in the exposure-only condition, each picture was always followed by a fixation cross. In the unrelated negative-label condition, each picture was always followed by a different unrelated negative word (e.g., a picture of a ferocious dog followed by the word "bomb" on one trial, the word "illness" on another trial, etc.). In the related negative-label condition, each picture was always followed by a different related negative word (e.g., a picture of a man assaulting a woman followed by the words "rape," "ruthless," etc.). Finally, in the neutral label condition, each picture was always followed by a different neutral (or slightly positive) related word (e.g., a picture of a deformed person followed by the words "body," "healing," etc.). Due to concerns about participant fatigue, we did not include a fourth condition of neutral unrelated labels Related and unrelated negative labels were included to test whether the semantic relationship between the affective label and the image made a difference in the long-term attenuation. We hypothesized that all negative labels, regardless of relevance to the image, would boost long-term attenuation of autonomic responding. As illustrated in Figure 1B, in the follow-up session (Day 8), there were five conditions: the same IAPS pictures as used in the four conditions in Day 1 but without any labels, plus a novel condition, in which never-before-seen negative IAPS pictures were presented, to test whether the long-term effects generalize to novel pictures.

\section{Materials}

The exposure session (Day 1) involved a total of 24 different negative IAPS pictures. Six different pictures were used for each of the four conditions. The four sets of pictures were equal in overall ratings of arousal and valence, as well as in thematic content. Each of the 24 pictures was presented six times, for a total of 144 trials. Throughout the exposure session, there were a total of 36 trials per condition. Consequently, there were 108 different words used (36 in each of the three labeling conditions). 

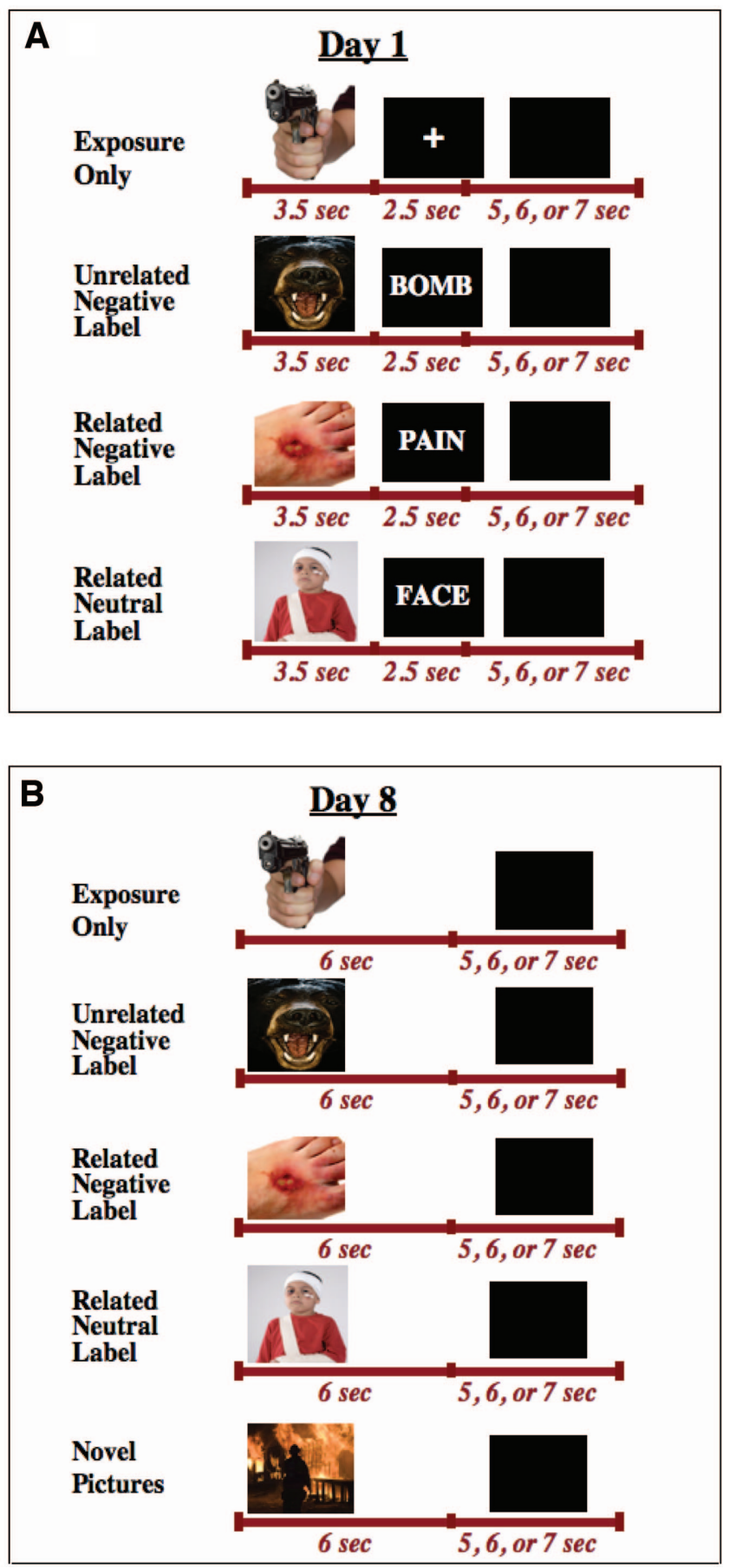

Figure 1. Diagram illustrating the design of Experiment 1. (A) On Day 1, participants were exposed to negative IAPS pictures under four different conditions (exposure alone, exposure plus unrelated negative labels, exposure plus related negative labels, and exposure plus neutral related labels). B) On Day 8, participants viewed the same pictures as Day 1 plus an additional set of never-before-seen (novel) pictures. (Note: Actual pictures used were more aversive than the ones depicted here. The pictures depicted in Figure 1 and Figure 4 were obtained from www.istockphoto.com.)
In the follow-up session (Day 8), the stimuli used were the same 24 negative IAPS pictures as in the training session, in addition to six novel negative IAPS pictures for the novel condition. The pictures in the novel condition had the same overall ratings of arousal and valence, as well as the same thematic content, as the pictures in each of the other conditions.

\section{Procedure}

Exposure session (Day 1). As illustrated in Figure 1A, each trial began with the presentation of a picture for $3.5 \mathrm{~s}$, followed by the text stimulus (a word or fixation cross) for $2.5 \mathrm{~s}$, followed by 5-7 s of a blank screen. Given that there were 144 trials and each trial lasted an average of $12 \mathrm{~s}$, the exposure session lasted approximately $29 \mathrm{~min}$, excluding the rest period between blocks and the 10-min adaptation and calibration period prior to data collection.

Before the start of the experiment, participants were told that they would see a number of pictures and words and that each picture would be shown several times. They were also told that although some of the pictures may be difficult to look at, they should try to keep their eyes on the picture while it is on the screen and allow themselves to emotionally respond it, and that if they see a word, they should read it silently to themselves.

Follow-up session (Day 8). As illustrated in Figure 1B, each picture was presented for $6 \mathrm{~s}$, followed by $5-7 \mathrm{~s}$ of a blank screen. Because there were 150 trials and each trial lasted an average of $12 \mathrm{~s}$, the follow-up session lasted approximately $22.5 \mathrm{~min}$. As before, participants were instructed to attend to each picture and to allow themselves to respond emotionally to each.

Psychophysiology protocol. Each participant was seated in a comfortable chair. A total of seven $\mathrm{Ag}-\mathrm{AgCl}$ reusable electrodes were attached with the use of adhesive collars: two on the nondominant hand for SCR, one below each clavicle, and one in the middle of the forehead for HR, and two above the left eyebrow for EMG. After the electrodes were attached but before the start of the experiment, participants remained seated for approximately 10 min for adaptation and calibration of the physiological recordings. The signals were acquired via Coulbourn Instruments and recorded with LabView software.

Peak SCR amplitude for each trial was scored by subtracting the valley point during the $0.5-4.0$-s period after picture onset from the peak point within the 6-s period after the valley point (Prokasy \& Kumpfer, 1973). Average HR during the 1-s period prior to the start of a trial served as the baseline HR measure for that trial. The initial deceleration phase was scored as the lowest HR during the first $2 \mathrm{~s}$ after picture onset minus baseline (Gatchel \& Lang, 1974). The deceleration component of HR is a distinct psychophysiological response to negative IAPS pictures compared to neutral or positive IAPS pictures (P. J. Lang, Greenwald, Bradley, \& Hamm, 1993).

Data analysis. The EMG data were discarded due to equipment failure. For each of the other two measures, a two (Day 1, Day 8) by four (exposure only, negative unrelated label, negative related label, neutral label) within-subjects analysis of variance (ANOVA) was conducted. The pairwise tests were calculated using a two-tailed $t$ test for the post hoc tests and a one-tailed $t$ test for the a priori hypotheses (Rosenthal \& Rosnow, 1991). Separate 
two-tailed tests were conducted for the tests of generalization to the novel condition.

\section{Results}

\section{$S C R$}

As indicated in Figure 2, SCR decreased from Day 1 to Day 8. A two-way (Day $\times$ Condition) within-subjects ANOVA of SCR indicated a significant main effect of Day, $F(1,15)=6.77, p<$ $.05, \eta^{2}=.31$, such that SCR was attenuated from Day 1 to Day 8 . Specifically, this attenuation occurred in the exposure-only, $t(17)=2.42, p<.05$; unrelated negative-label, $t(16)=4.23, p<$ .0005 ; and related negative-label, $t(18)=2.74, p<.01$ conditions, but not the neutral-label condition, $t(18)=1.11, p=.14$. There was no main effect of Condition on SCR, $F(3,45)=1.77, p=.17$, $\eta^{2}=.11$. However, there was a significant interaction between Day and Condition, $F(3,45)=4.08, p<.05, \eta^{2}=.21$.

Importantly, on Day 8, SCR to pictures from the unrelated negative-label condition was lower compared to pictures from the exposure-only, $t(19)=1.88, p<.05$; related negative-label, $t(19)=2.81, p<.05$; and neutral-label, $t(18)=3.40, p<.005$ conditions. Thus, although exposure led to reduced SCR from Day 1 to Day 8 in three of four conditions, and showed a trend toward significant SCR reduction in the fourth condition (neutral label), exposure plus unrelated negative words led to a greater reduction than exposure in any of the other conditions. (See Table 1 for additional statistics.)

\section{HR Deceleration}

As indicated in Figure 3, some evidence of enhanced attenuation of autonomic responding in the affect-label conditions was observed in the form of reduced HR deceleration. A two-way (Day $\times$
Table 1

Pairwise Comparisons of SCR in Experiment 1

\begin{tabular}{lrc}
\hline \multicolumn{1}{c}{ Comparison } & $t$ value & Significance \\
\hline Day 1 & & \\
Exposure only versus unrelated negative label & -0.68 & $n s$ \\
Exposure only versus related negative label & -1.37 & $n s$ \\
Exposure only versus neutral label & 0.89 & $n s$ \\
Unrelated negative label versus related & & \\
$\quad$ negative label & -0.48 & $n s$ \\
Unrelated negative label versus neutral label & 1.43 & $n s$ \\
Related negative label versus neutral label & 2.13 & $*$ \\
Day 8 & & \\
Exposure only versus unrelated negative & & \\
$\quad$ label` & 1.88 & $*$ \\
Exposure only versus related negative label^ & -1.94 & $n s$ \\
Exposure only versus neutral label & -2.12 & $*$ \\
Unrelated negative label versus related & & \\
$\quad$ negative label & -2.81 & $*$ \\
Unrelated negative label versus neutral label & -3.40 & $* *$ \\
Related negative label versus neutral label & -0.02 & $n s$ \\
Novel versus exposure only & 2.76 & $*$ \\
Novel versus unrelated negative label & 3.52 & $* *$ \\
$\quad$ Novel versus related negative label & 1.25 & $n s$ \\
Novel versus neutral label & 1.69 & $n s$ \\
\hline
\end{tabular}

Note. A negative $t$ value indicates that the mean in the second condition in the comparison is larger than the mean in the first condition. ${ }^{\wedge}$ indicates a priori hypothesis. $n s$ indicates not significant.

*Indicates significance at $p<.05 .{ }^{* *}$ Indicates significance at $p<.005$.

Condition) ANOVA of HR deceleration indicated no main effect of Day, $F(1,22)=1.76, p>.20, \eta^{2}=.07$, or Condition, $F(3,66)=.31, p>.20, \eta^{2}=.01$. However, pairwise comparisons indicated a reduction in HR deceleration from Day 1 to Day 8 in the unrelated negative-label, $t(22)=1.74, p<.05$, and related

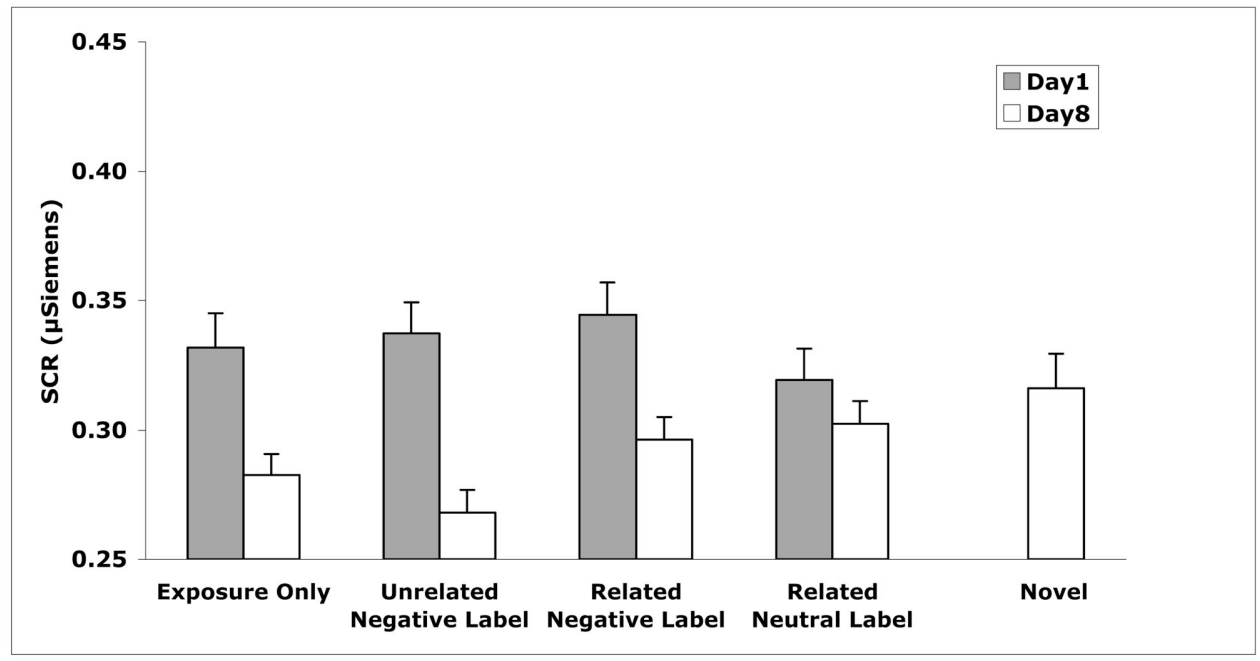

Figure 2. Skin conductance response as a function of day and exposure condition in Experiment 1. Higher bars indicate greater reactivity. Error bars indicate standard error. Unrelated negative labels produced the greatest long-term attenuation. Specifically, SCR decreased from Day 1 to Day 8 most reliably in the unrelated negative-label condition $(p<.005)$. On Day 8, SCR was lower in the unrelated negative-label condition compared to the exposure-only $(p<.05)$, related negative-label $(p<.05)$, and neutral-label $(p<.005)$ conditions. 


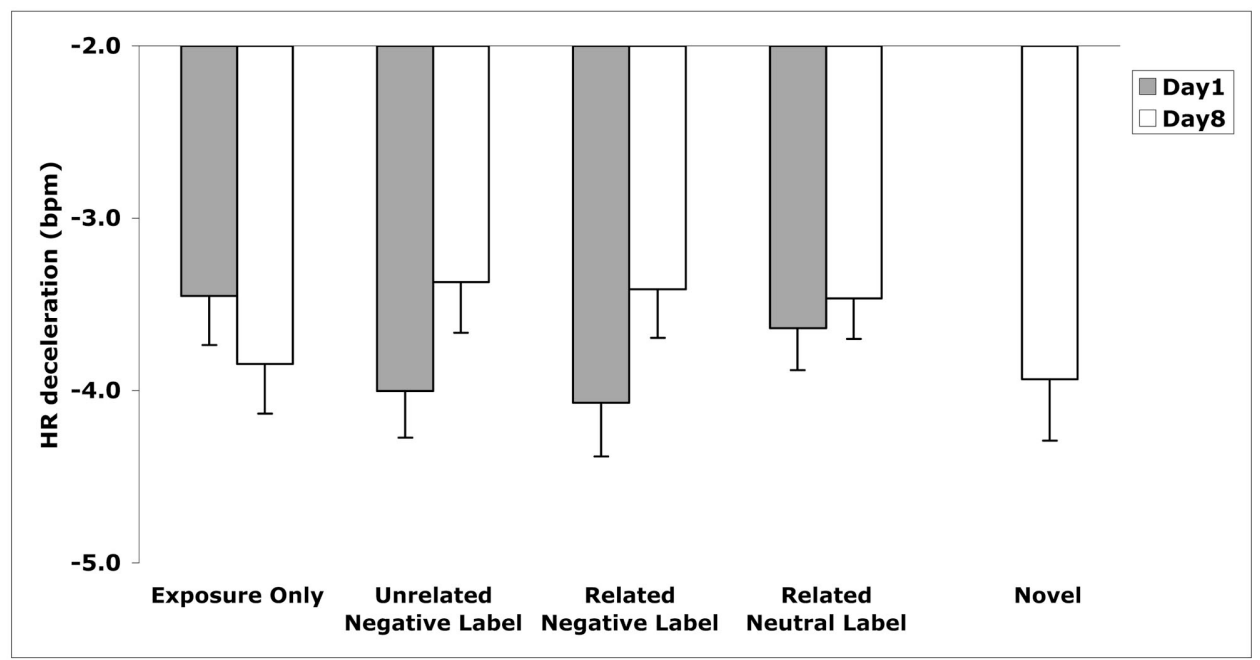

Figure 3. Heart rate deceleration as a function of day and exposure condition in Experiment 1. Greater negative scores indicate greater reactivity. Error bars indicate standard error. Negative labels produced the greatest long-term attenuation. Specifically, HR deceleration decreased from Day 1 to Day 8 most reliably in the unrelated negative-label $(p<.05)$ and related negative-label $(p<.05)$ conditions. On Day 8 , HR deceleration was lower in the unrelated negative-label condition compared to the exposure-only condition $(p<.05)$.

negative-label, $t(22)=2.18, p<.05$ conditions, but not in the exposure-only, $t(22)=-1.16, p>.20$, and neutral-label, $t(22)=$ $0.47, p>.20$, conditions. Furthermore, there was a significant interaction between Day and Condition, $F(3,66)=2.95, p<.05$, $\eta^{2}=.12$.

Importantly, although on Day 1 there was less HR deceleration in the exposure-only condition than the unrelated negativelabel, $t(22)=2.28, p<.05$, and related negative-label, $t(22)=$ $3.05, p<.01$, conditions, the reverse pattern was observed on Day 8; that is, on Day $8 \mathrm{HR}$ deceleration was reduced in the unrelated negative-label condition, $t(23)=1.82, p<.05$, and marginally reduced in the related negative-label condition, $t(23)=1.36, p<.10$, compared to the exposure-only condition. No other conditions differed from each other on Day 8. These results indicate that both related and unrelated negative labels may improve the effect of exposure, reducing HR deceleration in the long-term.

\section{Generalization}

There was no generalization to the novel pictures on Day 8 . Specifically, SCR in the novel condition (Day 8) was not different compared to any of the Day 1 conditions. Furthermore, comparing to other conditions on Day 8, SCR in the novel condition was higher than that in the exposure-only, $t(20)=2.76, p<.05$, and unrelated negative-label, $t(19)=3.52, p<.005$, conditions, and no different than that in the related negative-label, $t(20)=1.25$, $p>.20$, and the neutral-label, $t(20)=1.69, p=.11$, conditions. Similarly, there was no evidence in support of generalization in HR deceleration. In fact, HR deceleration was greater in the novel condition compared to the Day 1 exposure-only condition, $t(22)=$ $2.25, p<.05$, and marginally greater compared to the Day 8 unrelated negative-label, $t(23)=1.87, p<.08$, related negativelabel, $t(23)=1.98, p<.07$, and neutral-label, $t(23)=1.91, p<$ .07 , conditions.

\section{Discussion}

As hypothesized, repeated presentations of an aversive picture along with a negatively valenced word on Day 1 led to a greater reduction in autonomic reactivity when the aversive picture was encountered again on Day 8 than repeated presentations of the aversive picture alone on Day 1. Skin conductance response was reduced from Day 1 to Day 8 in the two negative-label conditions and the exposure-only condition, but not in the neutral-label condition, whereas HR deceleration was reduced in the two negativelabel conditions only. Critically, both SCR and HR deceleration were lower on Day 8 to pictures that had previously been exposed with unrelated negative labels than pictures that had been exposed alone. Together these results suggest that although exposure alone leads to long-term attenuation of autonomic responding, exposure plus unrelated negative words leads to greater attenuation than exposure alone. Neutral words did not enhance attenuation and, in the case of SCR, may have actually interfered with attenuation. Finally, there was no generalization of exposure effects to the novel pictures; reactivity to these pictures was at least as great as reactivity to the other pictures on Day 1 and was higher than reactivity to some of the pictures on Day 8 .

It is not clear why HR deceleration did not attenuate in the exposure-only condition. It appears that on Day $1 \mathrm{HR}$ did not decelerate to the pictures in the exposure-only condition compared to pictures in the unrelated negative-label or related negative-label conditions. It may be that the anticipation of an upcoming word led to additional HR deceleration in the two negative-label conditions (see Thayer and Lane, 2000). However, it is unclear why such anticipatory HR deceleration would not occur in the neutral-label condition. Therefore, the results of HR deceleration should be interpreted with caution.

Also unexpected was the result that unrelated negative words led to better long-term attenuation than related negative words in SCR. Given that on Day 1 SCR was the same to pictures in the two 
conditions, the Day 8 differences are not likely due to differential levels of emotional processing (Rachman, 1980) during the picture presentation on Day 1. The differential effect of related and unrelated negative words on Day 8 reactivity may instead be due to differential cognitive processing on Day 1 that may not necessarily be reflected by SCR in this task, or it may be due to differential processing during the 1-week period between the two experimental sessions. One possibility is that related words provide a means by which participants can ruminate about the pictures during the 1-week interim. Past research has shown that words can be used to induce rumination (Lyubomirsky, Caldwell, \& Nolen-Hoeksema, 1998) and that postevent rumination can disrupt emotional processing and lead to maintained anxiety (Abbott \& Rapee, 2004).

Unrelated words, on the other hand, may promote more elaborate processing of the pictures, such that participants may be thinking more deeply about each picture, in an attempt to find a relationship between it and the label. Such deeper processing may enhance exposure effects at follow-up (Rachman, 1980). That is, unrelated words may require an extra step or level of abstract processing that the related words do not. Research has shown that abstract, rather than concrete, construals of a negative experience mediate decreased negative emotions, presumably because they facilitate more cognitive processing (Kross, Ayduk, \& Mischel, 2005). This work may seem inconsistent with studies associating decreased symptom severity in posttraumatic stress disorder with increased coherence of the narrative the patient construes about the traumatic experience (Halligan, Michael, Clark, \& Ehlers, 2003). However, it remains to be determined whether abstract thinking or unrelated words provoke construal of an organized and coherent narrative.

It should also be noted that there is precedence for irrelevant linguistic processing to produce greater benefits than relevant linguistic processing. Verbal disclosure of traumatic, but not mundane, experience leads to improved well-being (Hemenover, 2003; Pennebaker, 1997), but interestingly, disclosing imagined traumatic experiences leads to better outcome, in terms of self-report depressed mood, fatigued mood, and avoidance, than disclosing real traumatic experiences (Greenberg, Wortman, \& Stone, 1996). Although it is still unclear why this phenomenon would occur, it appears to be a reliable effect.

\section{Experiment 2: Spider-Fearful Participants}

\section{Method}

\section{Participants}

Forty-eight college students (31 females; average age 19.3) who were fearful of spiders were selected from an undergraduate introductory psychology class at UCLA, using the 31-item Spider Phobia Questionnaire (SPQ), on which a score of 18 and above indicates moderate to severe fear of spiders (Klorman, Hastings, Weerts, Melamed, \& Lang, 1974). All participants gave informed consent. Individuals with heart, respiratory, or neurological problems or those on psychotropic medications were excluded. Participants were randomly assigned to one of three groups: exposure only, exposure plus negative unrelated labels (negative label), and exposure plus nonnegative labels (neutral label). There were 15 participants in the exposure-only group, 17 participants in the negative-label group, and 16 participants in the neutral-label group. The mean SPQ scores of the three groups (exposure only = 22.1 , negative label $=21.2$, neutral label $=21.9$ ) and the mean ages (exposure only $=19.4$, negative label $=19.1$, neutral label $=$ 19.3) were not significantly different from each other.

In the negative-label and neutral-label groups, the males and females did not differ in SCR; however, in the exposure-only group, the female participants' SCR was significantly greater than that of the male participants $(p<.05)$. To correct for gender differences in SCR, the statistical analyses of SCR covaried out gender effects. There was no significant effect of gender on HR acceleration.

\section{Design}

In this mixed-design experiment, the variable Day was within subjects, and the manipulation of linguistic processing was between groups. Unlike Experiment 1, in this experiment we did not manipulate labeling as a within-subjects variable, because all the pictures in this study depicted the same object (i.e., spiders), and it was unlikely that participants would show enhanced attenuation to pictures in one condition relative to pictures in another condition.

As illustrated in Figure 4A, in the exposure session (Day 1) each picture was always followed by either a fixation cross (exposureonly group), a different unrelated negative word (negative-label group), or a different related nonnegative word (neutral-label group). We used unrelated negative labels, because these labels led to the most long-term attenuation in Experiment 1. As illustrated in Figure 4B, in the follow-up session (Day 8), the same spider pictures as in the exposure session were used (exposed pictures), in addition to a novel set of spider pictures (novel pictures), to test for generalization of the attenuated response.

\section{Materials}

All groups viewed the same set of spider pictures. Twenty-four different pictures of spiders and tarantulas were used. Half of these were presented on both Days 1 and 8 (exposed pictures) and half were presented on Day 8 only (novel pictures). On Day 1, the 12 pictures were presented six times each, for a total of 72 trials. Thus 72 different negative words (e.g., "cancer," "war," "bullet") and 72 different neutral or slightly positive words (e.g., "little," "pet," "living") were used.

\section{Procedure}

Exposure session (Day 1). The exposure session consisted of 72 trials, divided into six 12-trial blocks. The trial structure was identical to Experiment 1 (see Figure 4). Because on average each trial lasted $12 \mathrm{~s}$, the exposure session lasted approximately 14.5 min. Before the start of the experiment, participants were told that they would see a number of spider pictures (and words, in the negative-label or neutral-label groups), and that each picture would be shown several times. They were also told that although some of the pictures may be difficult to look at, they should try to keep their eyes on the pictures while they are on the screen and allow themselves to respond emotionally to each. Participants in the negative-label and neutral-label groups were also told that if they see a word, they should read it silently to themselves. 

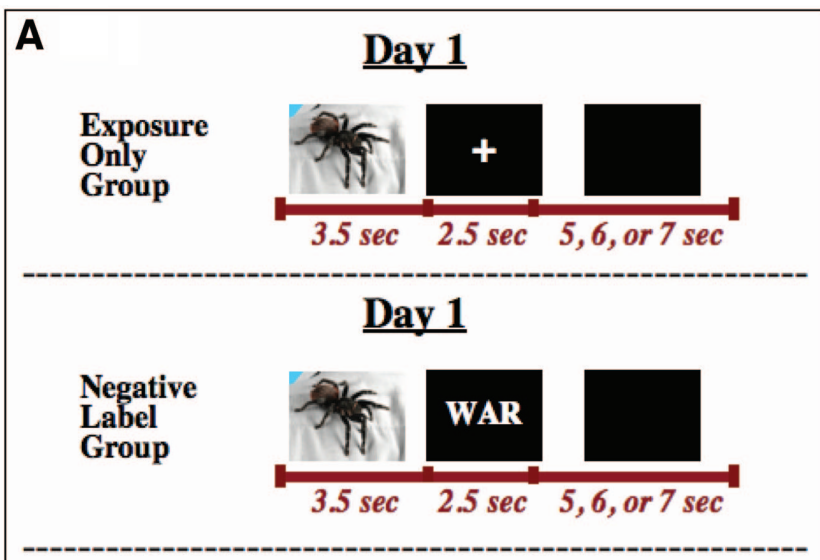

Day 1
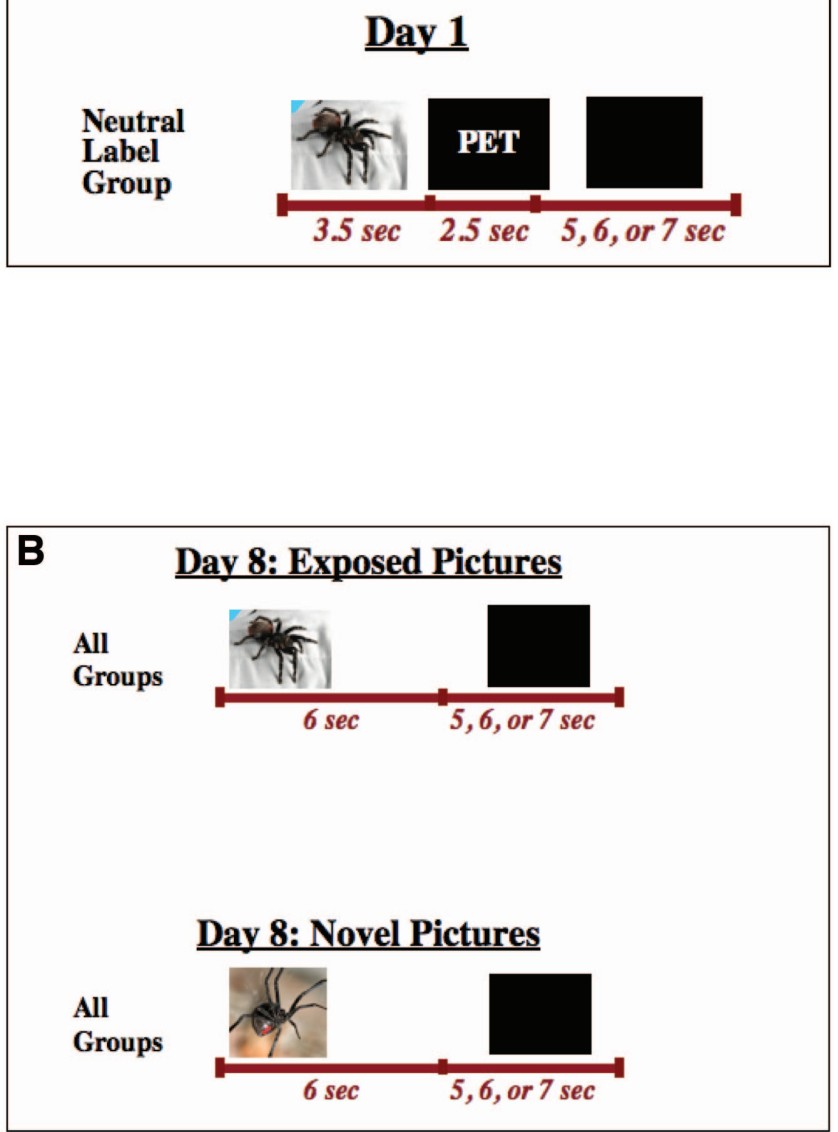

Figure 4. Diagram illustrating the design of Experiment 2. (A) On Day 1, spider-fearful individuals were exposed to spider pictures under one of three conditions (exposure alone, exposure plus negative labels, or exposure plus neutral related labels). (B) On Day 8, they viewed the same pictures as Day 1 plus an additional set of never-before-seen (novel) pictures.

Follow-up session (Day 8). The follow-up session consisted of 120 trials, divided into five 24-trial blocks. Each block consisted of the 12 previously exposed pictures and 12 novel spider pictures. As illustrated in Figure 4B, each picture was presented for $6 \mathrm{~s}$, followed by 5-7 s of a blank screen. Because on average each trial lasted $12 \mathrm{~s}$, the follow-up session lasted approximately $24 \mathrm{~min}$. As before, participants were instructed to attend to each picture and to allow themselves to respond emotionally to each.

Psychophysiology protocol. The psychophysiology protocol was the same as Experiment 1, except that for the HR measure, we scored the acceleration, rather than the initial deceleration, phase. Although overall HR decelerates during the 6-s presentation period more in response to negative IAPS pictures than to neutral or positive IAPS pictures (P. J. Lang et al., 1993), it accelerates in phobic individuals in response to phobia-related pictures (e.g., P. J. Lang, Melamed, \& Hart, 1970). The acceleration phase was scored by subtracting the baseline from the fastest HR subsequent to the initial deceleration and within the first $5 \mathrm{~s}$ after picture onset (Gatchel \& Lang, 1974).

\section{Results}

\section{$S C R$}

As indicated in Figure 5, SCR results were consistent with Experiment 1. A two-way (Group $\times$ Day) mixed-design ANOVA of SCR indicated a significant main effect of Day on SCR, $F(1,44)=23.92, p<.00005, \eta^{2}=.35$, such that SCR was attenuated from Day 1 to Day 8 . Specifically, this attenuation occurred reliably in the negative-label, $t(16)=4.75, p<$ .0005 , and exposure-only, $t(15)=1.97, p<.05$, groups, and marginally in the neutral-label group, $t(13)=1.63, p<.07$. There was no main effect of Group, $F(2,44)=.65, p>.20$, $\eta^{2}=.03$. However, there was a significant interaction between Day and Group, $F(2,44)=4.08, p<.05, \eta^{2}=.16$.

Importantly, on Day 8, SCR in the negative-label group was lower compared to the exposure-only group, $t(32)=2.61, p<.05$, and marginally lower compared to the neutral-label group, $t(30)=$ $1.70, p=.10$. The neutral-label group did not significantly differ from the exposure-only, $t(30)=.57, p>.20$, group on Day 8; nor did any group significantly differ from another on Day 1 . Thus, although all three exposure manipulations reduced SCR from Day 1 to Day 8 , exposure plus unrelated negative words led to a greater reduction.

\section{HR Acceleration}

A two-way ANOVA of HR acceleration indicated no main effect of Day, $F(1,39)=.03, p>.10, \eta^{2}=.001$, no main effect of Group, $F(2,39)=1.00, p>.10, \eta^{2}=.05$, and no interaction between Day and Group, $F(2,39)=.57, p>.10, \eta^{2}=.03$. Post hoc analyses also indicated no effects in HR deceleration.

\section{Generalization}

Comparisons of reactivity to the novel pictures on Day 8 with reactivity to the exposed pictures on Day 1 and Day 8 indicated a generalization to the novel pictures. Skin conductance response was not different to the novel pictures compared to the exposed pictures on Day 8 in the exposure-only, $t(15)=.43$, $p>.20$; neutral-label, $t(14)=.48, p>.20$; or the negativelabel groups, $t(15)=.29, p>.20$. Furthermore, in the negativelabel group, SCR to the novel pictures was lower compared to the exposed pictures on Day $1, t(15)=3.52, p<.005$; this effect was marginally significant in the neutral-label group, $t(13)=1.79, p<.10$, and not significant in the exposure-only 


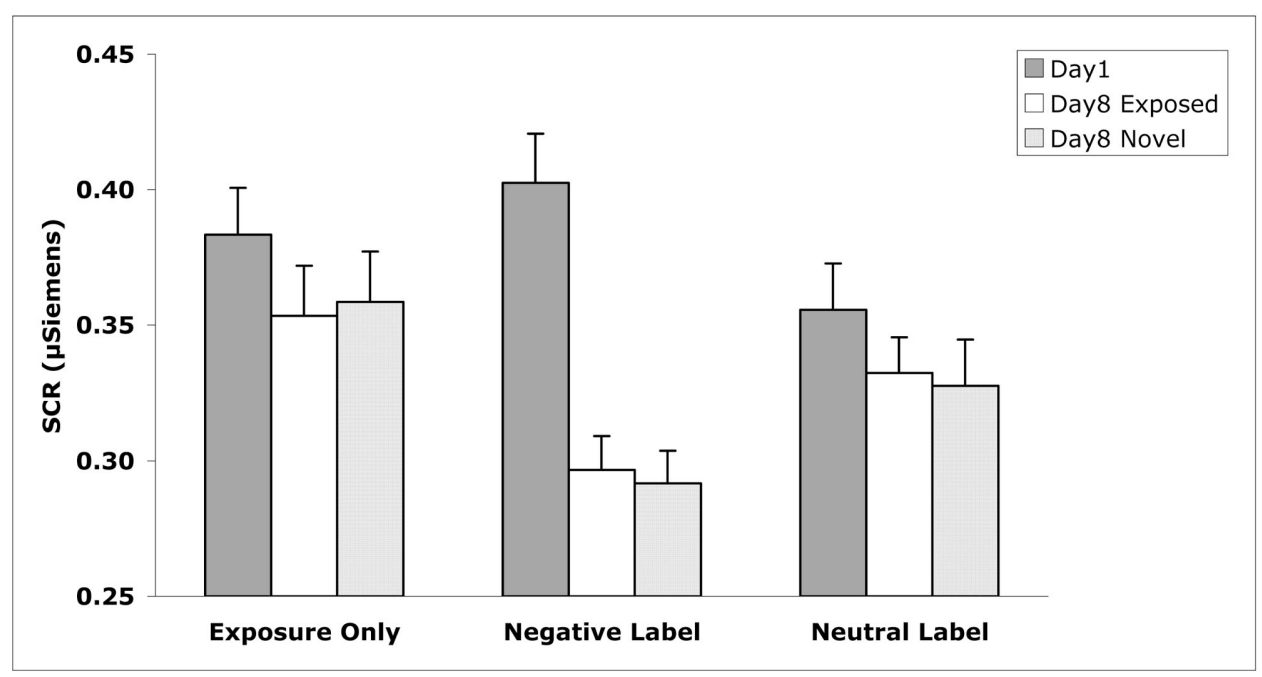

Figure 5. Skin conductance response as a function of day and exposure group in Experiment 2. Higher bars indicate greater reactivity. Error bars indicate standard error. The negative-label group showed the greatest long-term attenuation. Specifically, SCR decreased from Day 1 to Day 8 most reliably in the negative-label group $(p<.0005)$. On Day 8 , SCR was lower in the negative-label group compared to the exposure-only group $(p<.05)$.

group, $t(15)=1.35, p=.20$. HR acceleration to the novel pictures did not differ from the Day 1 or Day 8 exposed pictures in any group.

\section{Discussion}

As hypothesized, repeated presentations of fear-relevant pictures along with the negative words on Day 1 led to greater reduction in reactivity to the same pictures on Day 8 , compared to repeated presentations of the pictures alone on Day 1. Skin conductance response was reduced from Day 1 to Day 8 in all groups. Critically, on Day 8, SCR was lower in the negative-label group than the exposure-only group. As in Experiment 1, these results suggest that although exposure alone leads to long-term attenuation of autonomic responding, exposure plus negatively valenced words leads to greater effects than exposure alone. Neutral words did not enhance the effects relative to exposure alone. Furthermore, unlike Experiment 1, there was generalization to the novel pictures; on Day 8 SCR to the novel pictures and the exposed pictures did not differ, and in the negative-label group, SCR to the novel pictures was reduced compared to Day 1.

It is not clear why no effects were found in HR acceleration or deceleration. However, discordance between SCR and HR measures is not uncommon, due to homeostatic constraints on HR (Öhman, 1987). It is also possible that affective words do not uniformly modulate the separate phases of HR, namely, initial deceleration (Experiment 1) and acceleration (Experiment 2), in the long term.

The fact that the exposure effects generalized to novel pictures suggests that this paradigm exposed a sufficient variety of aversive stimuli in the exposure session to allow for learning to be broadly applicable (A. J. Lang, Craske, \& Bjork, 1999), at least to the extent that the stimuli are pictures. Furthermore, given that the generalization was best in the negative-label group, an additional advantage of adding negative words to exposure may be that it enhances generalization. No generalization was observed in the Experiment 1, perhaps because the aversive stimuli belonged to a wide range of categories and not enough variety was presented for each category, due to the use of a within- rather than betweensubjects design. Because the studies also varied in the samples (fearful vs. nonselected population), future work is necessary to isolate the variable(s) promoting generalization.

\section{General Discussion}

In two experiments, exposure to threatening pictures along with unrelated negative words produced a greater reduction in longterm autonomic reactivity to those pictures than exposure alone. In Experiment 1, in an unselected sample, SCR to previously exposed aversive IAPS pictures was attenuated a week later in a follow-up session. However, both SCR and HR deceleration at follow-up were reduced to pictures that were earlier viewed with unrelated negative labels compared to pictures that were viewed with a fixation cross, and SCR was also reduced compared to pictures that were viewed with related negative labels or neutral labels. Similarly, in Experiment 2, in a sample of spider-fearful individuals, SCR to previously exposed spider pictures was attenuated a week later in a follow-up session. However, at follow-up, SCR was reduced in the group who earlier viewed the pictures with unrelated negative labels compared to the exposure-only group. Together these results suggest that exposure alone attenuates autonomic reactivity to aversive pictures, but that exposure plus unrelated negative labels enhances this attenuation. Finally, this attenuation generalized to novel pictures in spider-fearful participants in Experiment 2 but not in the nonselected participants in Experiment 1.

The finding that negatively valenced words were more effective than neutral words is consistent with the fMRI finding that amyg- 
dala activation to evocative pictures decreases in the presence of negative but not neutral words (Lieberman, Eisenberger et al., 2007). According to this and other similar studies (e.g., Hariri et al., 2000; Lieberman, Crockett et al., 2007; Lieberman et al., 2005), emotional reactivity to evocative pictures is dampened while one engages in linguistic processing of those pictures (see also Borkovec et al., 1998). Here we show for the first time that negative words can also have a lasting (1 week) effect on emotional reactivity. In the presence of negative words, aversive stimuli may be processed in a deeper and more symbolic manner, allowing the ameliorative effect of the words on emotional reactivity to last long after the words have disappeared. Previous work has shown that emotion regulation may produce a rebound effect, leading to poorer mood and performance subsequent to the regulation, because such effortful processes deplete cognitive and self-regulatory resources (Baumeister, Bratslavsky, Muraven, \& Tice, 1998; Wegner, 1994). The current paradigm, however, did not require any intentional self-regulation. Thus, negative words may allow a different form of emotion regulation that in some cases may have beneficial long-term effects, particularly when the words are used in a way that does not interfere with emotional processing (i.e., introduced after initial exposure).

Some may argue that the more arousing and salient nature of negative words relative to neutral words may also play a role in their long-term effect. Animal studies have shown that extinction is enhanced when a "concurrent excitor," another arousing stimulus in addition to the to-be-extinguished stimulus, is present during exposure (Rescorla, 2000; Thomas \& Ayres, 2004). Increased arousal during extinction learning, by administration of a noradrenergic agonist (Cain, Blouin, \& Barad, 2004) or glucocorticoids (Soravia et al., 2006), may also enhance extinction learning. Thus, negative and unrelated words may provide some additional arousal that enhances the effects of exposure to aversive stimuli. Given that in both experiments, SCR on Day 1 was not different in the exposure-only and the negative-label conditions, it is unlikely that the enhanced effects at Day 8 in the negative-label conditions were due to increased sympathetic arousal during exposure to the pictures on Day 1. However, the current study design does not allow a measure of arousal in response to the words. It may be the case that high-arousal words facilitate deeper and more complete processing relative to low-arousal words or no words.

Exposure treatment for anxiety disorders has been in large part modeled after experimental findings of classical fear conditioning and extinction in nonhuman animals, particularly rodents. Extinction of fear in humans seems to follow some of the same learning principles (Davey, 1992) and relies on similar neural systems (Delgado, Olsson, \& Phelps, 2006) as extinction in rodents. However, unlike rodents, humans have the capacity to think symbolically and use language to navigate through the challenges of daily life. Classical conditioning studies in humans have demonstrated that verbally transmitted information alone can reduce the expression of conditioned fear (Davey \& McKenna, 1983; Delgado et al., 2004; but also see Davey, 1992). However, until now no study had examined the effect of language on the outcome of exposure treatment. Although the current study utilized only a rudimentary form of exposure treatment, it demonstrates for the first time that affective language facilitates exposure-related attenuation of autonomic reactivity to aversive and fear-relevant stimuli.
Some limitations of the current study warrant mention. First, because we did not collect self-report measures of affect, we do not know whether subjective indices of fear or aversion were also reduced after exposure. However, in another study of spiderfearful individuals involving repeated presentations of visual images of spiders, both SCR and subjective ratings of fear were reduced from pre to postexposure (Vansteenwegen et al., in press). Second, because we did not find consistent effects in HR across experiments, the autonomic effects discussed here cannot be generalized beyond sympathetic arousal. Furthermore, although the current study demonstrates enhanced exposure effects with affective labels 1 week after treatment, it remains to be determined whether this effect is maintained over longer intervals of time. Additionally, given that verbal communication during therapy does not typically consist of reading single words, follow-up studies need to be conducted with complete sentences, read out loud by the participant, or presented in auditory form, to simulate a therapist's voice. Similarly, clinical trials would need to be conducted to determine whether the addition of negative language to exposure in vivo can also enhance treatment outcome.

The way linguistic stimuli were used in the current experiments is distinct from the way language is used in cognitive therapy to reduce fear and anxiety. In cognitive therapy, language is used before and during therapy to help create a calm environment (see Thorpe \& Salkovskis, 1997). In the current experiments, however, words were introduced after initial exposure to allow emotional reactivity to develop, at least initially. Furthermore, in cognitive therapy language is used to shift appraisals of feared stimuli away from threatening to benign or nonnegative. In our study, the type of language most beneficial was negative and unrelated to the content of the aversive stimulus. These discrepancies raise the need for future studies that specifically compare the current approach with cognitive therapy.

Although the procedures utilized in this study do not closely model any particular form of psychological therapy, they provide a rudimentary analogue of exposure treatment with and without the use of language. Some form of "talk therapy," particularly cognitive therapy, is typically incorporated into the treatments for fear and anxiety. Empirical tests of whether the incorporation of cognitive therapy into exposure therapy for phobias improves outcome of exposure therapy have not been consistent; some indicate an added value of cognitive therapy and some do not (Craske, 1999; Craske \& Rowe, 1997). A potential explanation for the inconsistent results is the many confounding variables that are present when comparing one treatment component (exposure) to another (cognitive therapy); these variables include treatment expectancy, treatment duration, and therapist-patient relationship variables. The current study introduces a novel paradigm that tests the role of verbal processing in the reduction of emotional responding in a well-controlled experimental manner. To the extent that cognitive therapy can be simulated with manipulation of simple sentences, read by the participant or presented in auditory form, then variants of this paradigm can be used for more controlled investigations of the effect of cognitive therapy on exposure outcome.

In conclusion, our results suggest that the effect of exposure therapy may be enhanced by use of unrelated negative language during treatment. It may thus be the case that cognitive therapy, or other talk therapy that accompanies exposure treatment, would be more effective in facilitating fear extinction to the extent that 
anxiety-provoking, rather than neutral language, is used. A broader implication of this line of research would be that perhaps part of the benefit of talk therapy is due to mere linguistic processing of aversive experiences. However, follow-up studies need to be conducted to explore these implications.

\section{References}

Baumeister, R. F., Bratslavsky, E., Muraven, M., \& Tice, D. M. (1998). Ego depletion: Is the active self a limited resource? Journal of Personality and Social Psychology, 74, 1252-1265.

Beauregard, M., Levesque, J., \& Bourgouin, P. (2001). Neural correlates of conscious self-regulation of emotion. Journal of Neuroscience, 21, 6993-7000.

Berntson, G. G., Cacioppo, J. T., \& Quigley, K. S. (1993). Cardiac psychophysiology and autonomic space in humans: Empirical perspectives and conceptual implications. Psychological Bulletin, 114, 296-322.

Borkovec, T. D., Ray, W. J., \& Stoeber, J. (1998). Worry: A cognitive phenomenon intimately linked to affective, physiological, and interpersonal behavioral processes. Cognitive Therapy and Research, 22, 561-576.

Brewer, W. F. (1974). There is no convincing evidence for operant and classical conditioning in humans. In W. B. Weimer \& D. J. Palermo (Eds.), Cognition and the symbolic processes. Hillsdale, NJ: Erlbaum.

Cain, C. K., Blouin, A. M., \& Barad, M. (2004). Adrenergic transmission facilitates extinction of conditional fear in mice. Learning and Memory, $11,179-187$

Center for the Study of Emotion and Attention, National Institute of Mental Health. (1999). The International Affective Picture System: Digitized photographs. Gainesville: University of Florida, The Center for Research in Psychophysiology.

Chambless, D. L., \& Hollon, S. D. (1998). Defining empirically supported therapies. Journal of Consulting Clinical Psychology, 66, 7-18.

Craske, M. G. (1999). Anxiety disorders: Psychological approaches to theory and treatment. Boulder: Westview Press.

Craske, M. G., \& Mystkowski, J. L. (2006). Exposure therapy and extinction: Clinical studies. In M. G. Craske, D. Hermans, \& D. Vansteenwegen (Eds.), Fear and learning: Basic science to clinical application (pp. 217-234). Washington, DC: American Psychological Association.

Craske, M. G., \& Rowe, M. K. (1997). A comparison of behavioral and cognitive treatments of phobias. In G. C. L. Davey (Ed.), Phobias: A handbook of theory, research and treatment (pp. 247-280). Chichester, United Kingdom: Wiley.

Critchley, H. et al. (2000). Explicit and implicit neural mechanisms for processing of social information from facial expressions: A functional magnetic resonance imaging study. Human Brain Mapping, 9, 93-105.

Davey, G. C. L. (1992). Classical conditioning and the acquisition of human fears and phobias: A review and synthesis of the literature. Advances in Behaviour Research and Therapy, 14, 29-66.

Davey, G. C. L., \& McKenna, I. (1983). The effects of postconditioning revaluation of CS1 and UCS following Pavlovian second-order electrodermal conditioning in humans. Quarterly Journal of Experimental Psychology, 35B, 125-133.

Delgado, M. R., Olsson, A., \& Phelps, E. A. (2006). Extending animal models of fear conditioning to humans. Biological Psychology, 73, $39-48$.

Delgado, M. R., Trujillo, J. L., Holmes, B. D., Nearing, K. I., LeDoux, J. E., \& Phelps, E. A. (2004). Emotion regulation of conditioned fear: The contributions of reappraisal. Poster session presented at the annual meeting of the Cognitive Neuroscience Society, San Francisco.

Eysenck, H. J. (1979). The conditioning model of neurosis. Behavioral and Brain Sciences, 2, 155-199.

Foa, E. B., \& Kozak, M. J. (1986): Emotional processing of fear: Exposure to corrective information. Psychological Bulletin, 99, 20-35.

Foa, E. B., \& McNally, R. J. (1996). Mechanisms of change in exposure therapy. In M. Rapee (Ed.), Current controversies in the anxiety disorders (pp. 329-343). New York: Guilford Press.

Gatchel, R. J., \& Lang, P. J. (1974). Effects of interstimulus interval length and variability on habituation of autonomic components of the orienting response. Journal of Experimental Psychology, 103, 802-804.

Gross, J. (1998). Antecedent and response focused emotion regulation: Divergent consequences for experience, expression, and physiology. Journal of Personality and Social Psychology, 74, 224-237.

Hariri, A. R., Bookheimer, S. Y., \& Mazziotta, J. (2000). Modulating emotional responses: Effects of a neocortical network on the limbic system. Neuroreport, 17, 43-48.

Hariri, A. R., Mattay, V. S., Tessitore, A., Fera, F., \& Weinberger, D. R. (2003). Neocortical modulation of the amygdala response to fearful stimuli. Biological Psychiatry, 53, 494-501.

Hemenover, S. H. (2003). The good, the bad, and the healthy: Impacts of emotional disclosure of trauma on resilient self-concept and psychological distress. Personality and Social Psychology Bulletin, 29, $1236-1244$

Klorman, R., Hastings, J. E., Weerts, T. C., Melamed, B. G., \& Lang, P. J. (1974). Psychometric description of some specific fear questionnaires. Behavior Therapy, 5, 401-409.

Lang, A. J., Craske, M. G., \& Bjork, R. A. (1999). Implications of a new theory of disuse for the treatment of emotional disorders. Clinical Psychology: Science and Practice, 6, 80-94.

Lang, P. J., Bradley, M. M., \& Cuthbert, B. N. (1999). International affective picture system (IAPS): Instruction manual and affective ratings. Gainesville: University of Florida, The Center for Research in Psychophysiology.

Lang, P. J., Greenwald, M. K., Bradley, M. M., \& Hamm, A. O. (1993). Looking at pictures: Affective, facial, visceral, and behavioral reaction. Psychophysiology, 30, 261-273.

Lang, P. J., Melamed, B. G., \& Hart, J. A. (1970). Psychophysiological analysis of fear modification using an automated desensitization procedure. Journal of Abnormal Psychology, 76, 220-234.

Levesque, J., Eugene, F., Joanette, Y., Paquette, V., Mensour, B., Beaudoin, G., Leroux, J. M., Bourgouin, P., \& Beauregard, M. (2003). Neural circuitry underlying voluntary suppression of sadness. Biological Psychiatry, 53, 502-510.

Lieberman, M. D., Crockett, M. J., Inagaki, T., \& Tabibnia, G. (2007). Affect labeling reduces emotional responding: Self-report and physiological evidence. Unpublished manuscript.

Lieberman, M. D., Eisenberger, N. I., Crockett, M. J., Tom, S. M., Pfeifer, J. H., \& Way, B. M. (2007). Putting feelings into words: Affect labeling disrupts amygdala activity to affective stimuli. Psychological Science, $18,421-428$

Lieberman, M. D., Hariri, A., Jarcho, J. M., Eisenberger, N. I., \& Bookheimer, S. Y. (2005). An fMRI investigation of race-related amygdala activity in African-American and Caucasian-American individuals. $\mathrm{Na}$ ture Neuroscience, 8, 720-722.

Ochsner, K. N., Bunge, S. A., Gross, J. J., \& Gabrieli, J. D. E. (2002). Rethinking feelings: An fMRI study of the cognitive regulation of emotion. Journal of Cognitive Neuroscience, 14, 1215-1229.

Öhman, A. (1987). The psychophysiology of emotion: An evolutionarycognitive perspective. Advances in Psychophysiology, 2, 79-127.

Öhman, A., Hamm, A. O., \& Hugdahl, K. (2000). Cognition and the autonomic nervous system: Orienting, anticipation, and conditioning. In J. T. Cacioppo, L. G. Tassinary, \& G. G. Berntson (Eds.), Handbook of psychophysiology (pp. 533-575). New York: Cambridge University Press.

Pavlov, I. P. (1927). Conditioned reflexes (G. V. Anrep, Trans.). London: Oxford University Press.

Pennekaber, J. W. (1997). Writing about emotional experiences as a therapeutic process. Psychological Science, 8, 162-166.

Prokasy, W. F., \& Kumpfer, K. L. (1973). Classical conditioning. In W. F. 
Prokasy \& D. C. Raskin (Eds.), Electrodermal activity in psychological research (pp. 157-202). San Diego: Academic Press.

Rachman, S. (1980). Emotional processing. Behaviour Research and Therapy, 18, 51-60.

Rescorla, R. A. (2000). Extinction can be enhanced by a concurrent excitor. Journal of Experimental Psychology: Animal Behavior Processes, 26, 251-260.

Soravia, L. M., Heinrichs, M., Aerni, A., Maroni, C., Schelling, G., Ehlert, U., Roozendaal, B., \& deq Uervain, D. J. (2006). Glucocorticoids reduce phobic fear in humans. Proceedings of the National Academy of Sciences of the USA, 103, 5585-5590.

Thayer, J. F. and Lane, R. D. (2000). A model of neurovisceral integration in emotion regulation and dysregulation. Journal of Affective Disorders, 61, 201-216.

Thomas, B. L., \& Ayres, J. J. B. (2004). Use of the ABA fear renewal paradigm to assess the effects of extinction with co-present fear inhibitors or excitors: Implications for theories of extinction and for treating human fears and phobias. Learning and Motivation, 35, $22-52$.

Thorpe, S. J., \& Salkovskis, P. M. (1997). Animal phobias. In G. C. L. Davey (Ed.), Phobias: A handbook of theory, research and treatment (pp. 247-80). Chichester: Wiley.

Titchener, E. B. (1908). Lectures on the elementary psychology of feeling and attention. New York: MacMillan.

Vansteenwegen, D., Vervliet, B., Iberico, C., Baeyens, F., Van den Bergh, O., \& Hermans, D. (2007). The repeated confrontation with videotapes of spiders in multiple contexts attenuates renewal of fear in spider anxious students. Behaviour Research and Therapy, 46, 1169-1179.

Wegner, D. M. (1994). Ironic processes of mental control. Psychological Review, 101, 34-52.

Received November 29, 2006

Revision received December 19, 2007

Accepted January 2, 2008

\section{ORDER FORM}

Start my 2008 subscription to Emotion

ISSN: $1528-3542$

\section{\$52.00, APA Member/Affiliate \$98.00, INDIVIDUAL NONMEMBER $\$ 400.00$, InSTITUTION In DC add $5.75 \%$ / In MD add 6\% sales tax Total Amount Enclosed}

$\$$

Subscription orders must be prepaid. (Subscriptions are on a calendar year basis only.) Allow 4-6 weeks for delivery of the first issue. Call for international subscription rates.

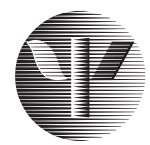

AMERICAN PSYCHOLOGICAL ASSOCIATION
SEND THIS ORDER FORM TO:

American Psychological Association Subscriptions

750 First Street, NE

Washington, DC 20002-4242

Or call 800-374-2721, fax 202-336-5568

TDD/TTY 202-336-6123.

For subscription information, e-mail: subscriptions@apa.org
Check enclosed (make payable to APA)

Charge my: $\bigcirc$ VISA $\bigcirc$ MasterCard $\underset{\text { American }}{\text { Express }}$

Cardholder Name

Card No. Exp. Date

\section{BILLING ADDRESS:}

Street

City State Zip

Daytime Phone

E-mail

\section{MAIL TO:}

Name

Address

City

State Zip

APA Member \# EMOA08 